\title{
The Various Software Automated Web Testing Tools
}

\author{
Dr Leelavathi Rajamanickam, \\ Senior Lecturer \\ ${ }^{1}$ School of Information Technology, SEGi University, MALAYSIA. \\ Email: leelavathiraj@segi.edu.my
}

\begin{abstract}
Software testing is an important part in software development process. We have a great number of software testing tools at our disposal for testing a software product. Complex systems are being built and testing throughout the software development cycle is valid to the success of the software. Testing is very expensive process. Manual testing involves a lot of effort, Measured in person per month. These efforts can be reduced by using the automated testing with specific tools.
\end{abstract}

Keywords: software testing, automated testing, manual testing

\section{0 introduction}

Software testing is about testing a feature with varying test data to get a result and then comparing the actual result with expected result, it is not merely finding defects or bugs in the software; it is the completely dedicated discipline of evaluating the quality of the software [1]. Software testing is a process is to identify all bugs that exist in a software product. It is the process of evaluating all the components of a system verifies that it satisfies specified requirements or to classify differences between expected and actual results. Software testing is also performed to achieving quality by using the software with applicable test cases. Testing can be integrated at various points in the development process depending upon the tools and methodology used. Software Testing usually starts after requirements [2]. At a unit level phase, it starts concurrently with coding; whereas at integration level, it starts when coding is completed. Testing process can be performed by two ways that are manual or automation. 


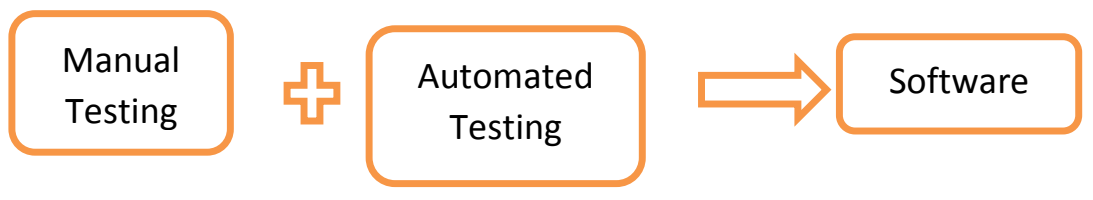

Figure 1: Software Testing

Manual testing is a process to test the software manually to find out the bugs. Manual testing is performed without using any automated tool. While performing the manual testing a test plan is used that describe the systematic and detailed approach of testing a software application. The goal of the testing is to make sure that the software application under test is defect free. Manual testing is not suitable for large projects as it requires more resources and time [4]. Automated testing is a process in which tools execute a pre-defined scripted test on software to find defects. Automated software testing is the finest way to increase the effectiveness and efficiency of software testing. Automation testing can does what manual testing does not. Automation testing also improves the accuracy and saves the time of the tester \& organization's money. It is best appropriate in the environment where the requirements are repeatedly changing $\&$ huge amount of regression testing is required to be performed.

\subsection{Automated Testing Process}

Test automation interface is a platform which provides a single workspace for incorporating multiple testing tools. To improve the efficiency and flexibility of maintaining test scripts test automation interface is use. It includes Interface Engine which consist runner to execute the test scripts, the Interface Environment that consists Framework and Project Library and Object Repository are collection of application object data. 


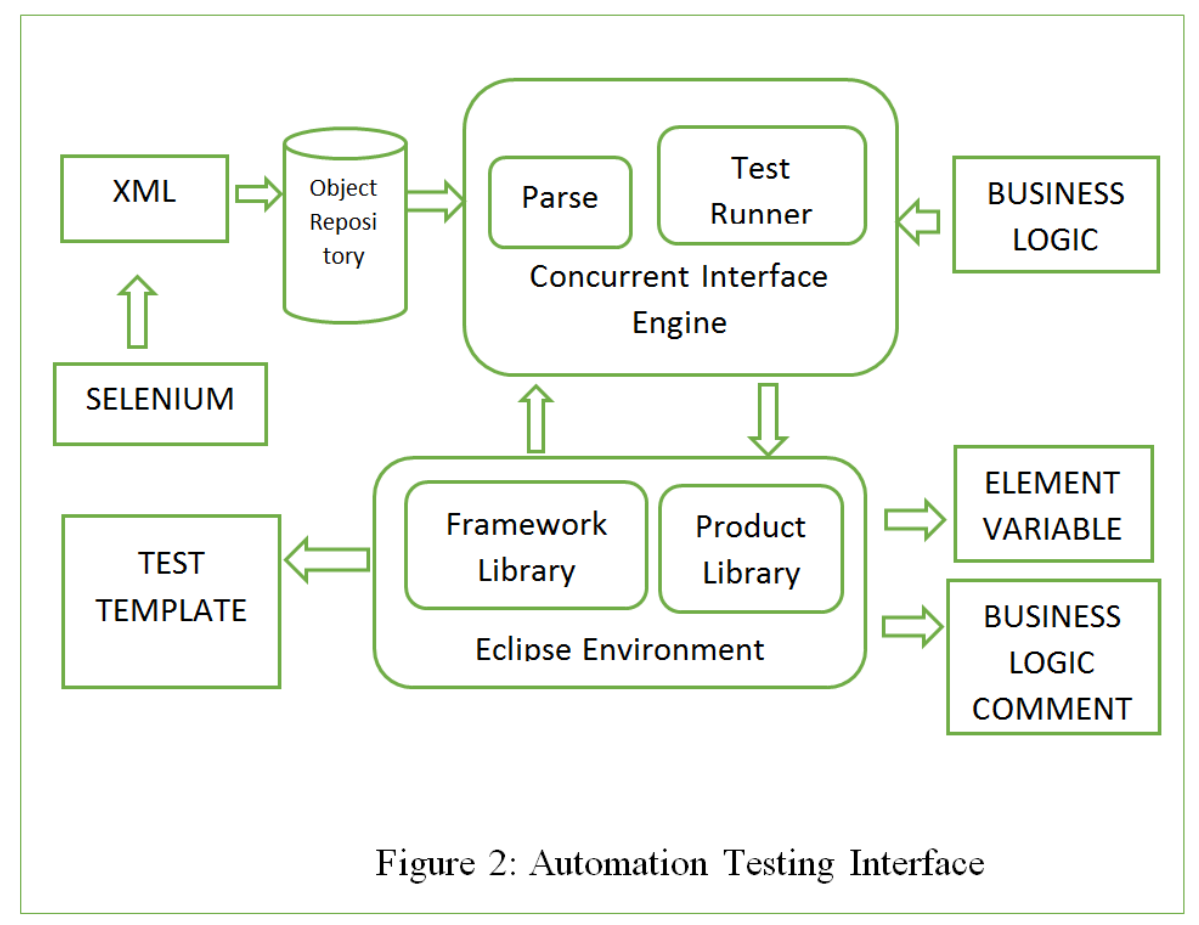

The automation software testing consists of a sequence of activities, processes and tools that processed in order to execute the test on software and to keep the record of the result of tests [7]. The following activities include in testing process:

- Test planning

- Test design

- Test Implementation

- Test execution

- Test evaluation

A general testing process is depicted in Figure 1:

Each activity has performed some tasks that are used by another phase. At the end, a bug reports will be documented. These documents are further used by the development team to recognize the cause of faults and to correct them. After elaborating the test plan analysis is performed on the requirements and test objectives are defined. The design phase is focused on the definition and test procedures. At this step it is decided that which part should be tested manually and which will be tested automatically [5]. General approaches that are used for test automation are: Code driven testing: the public interfaces to libraries, classes and modules are tested with a number of inputs to validate that the results are correct. Graphical 
User Interface: Keystrokes and mouse clicks interface events are generated by a testing framework and detect the changes to validate that the observable performance of program is correct.

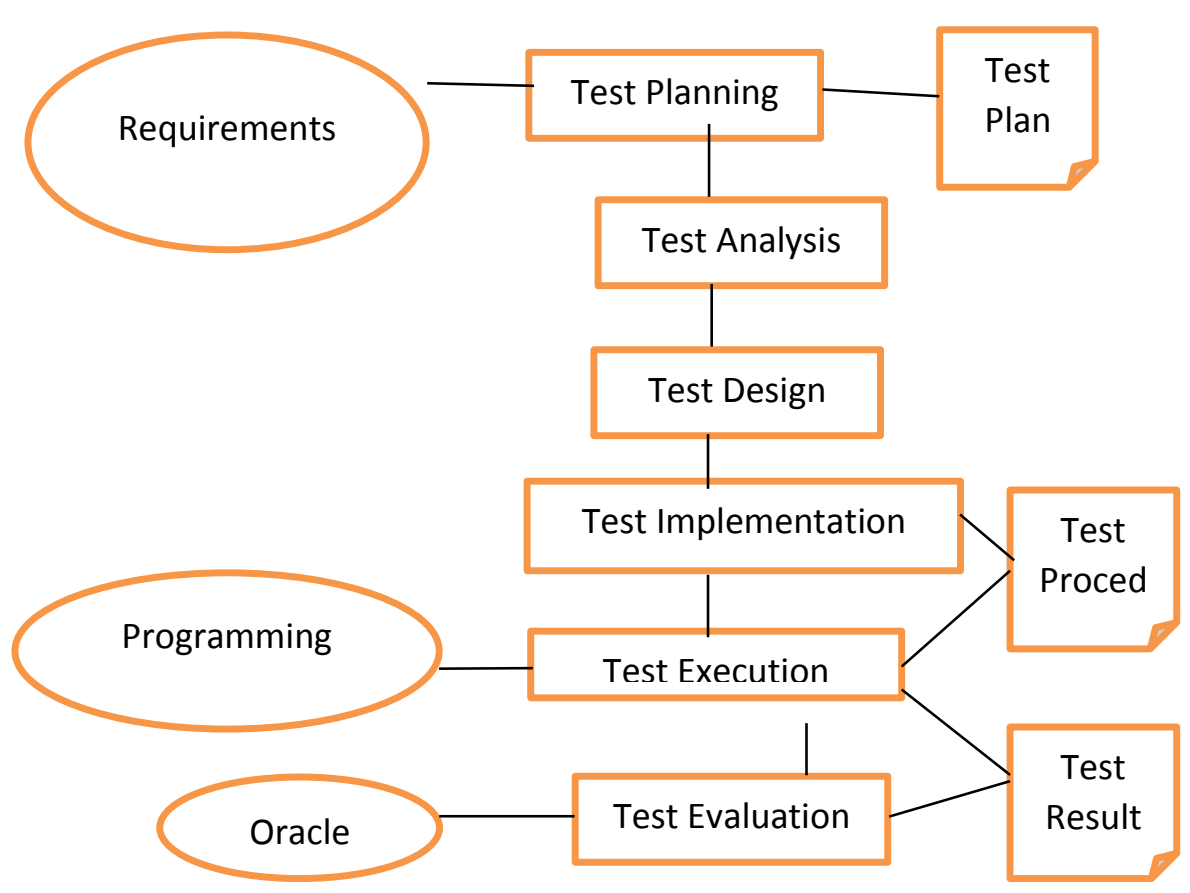

Figure 3: Testing Process

\subsection{Software Automated Web Testing Tools}

Various types of tools are used for automated web testing and they can be used in different areas of testing. The selection of tool is based on the type of application which we want to test like automated web testing tools, GUI testing tools [8].

\section{Ranorex}

Ranorex allows you to automate your web application testing (among other things) and both record user interactions and play them back to execute your tests. Ranorex is one of the more popular commercial tools to build and run automated web and GUI tests. It is a GUI test automation framework which provides testing of a wide range of 
desktop, web and mobile applications. Test cases once written can be executed on different platforms. It simulates the user actions by record and replay tool into recording modules. Ranorex is easy to use and affordable even for small testing teams.

\section{Test Complete}

Test complete has open flexible architecture for maintaining and executing automated tests for web. This tool helps to keep the balance between quality and speed of delivery of applications at affordable cost. With test complete tool different types of testing can be done like unit testing and GUI testing, regression testing etc

\section{Selenium}

Selenium is a popular automated web testing tool and helps you automate web browsers across different platforms. Selenium has the support of some of the largest browser vendors who have taken steps to make Selenium a native part of their browser.

Selenium is an open source web testing tool which is used to test the web browsers across different platforms. It is divided into four components: First is, Selenium IDE which is used as a prototyping tool and no programming language is required. Second Selenium Remote Control that allow users to use the programming language. Third Web Driver which implement a stable approach by direct communication between the test scripts and browsers. Forth, Selenium Grid that helps to execute parallel tests on different browsers by using with Selenium Remote Control.

\section{Sahi}

Sahi is a tool for automation of web application testing. Sahi is available as a free open source edition as well as a commercial Pro edition. This tool is used by the developers for fixing and reproducing bugs, QAs for functional testing and by business analysts for defining and verifying functionality. It supports java script language and offers easily editable scripts.

\section{Watir}


Watir is a set of Ruby libraries for automating web browsers and allows you to write tests that are easy to read and maintain. Watir drives browsers the same way people do (it clicks links, fills in forms, presses buttons etc.) and also checks results such as whether expected text appears on the page. Watir is an open source tool for automating web browsers. This tool is simple and flexible in terms of easy to read and maintain. It supports only Internet Explorer, Firefox and Opera. It also supports multiple browsers on different platforms.

\section{Tellurium}

Tellurium is a web automation tool that allows you to design and write your automated tests using plain English without any scripting or programming experience. Tellurium is an open source automated testing framework for testing web applications. It was developed from Selenium framework with different testing concept. It is built with UI module concept which helps to write reusable and easily maintainable tests.

\section{Windmill}

Windmill is a web testing tool designed to help testers automate and debug web applications. It comes with a cross-browser test recorder, JavaScript integration and an interactive shell to automate web browsers. Windmill is a web testing framework that provides automation testing and debugging capabilities. The purpose of windmill is to create test writing portable and easier. It supports Firefox, Safari, Chrome and Opera browser. The tool runs on Microsoft Windows, Linux and Mac OS X. Without require any programming language Windmill provides a cross-browser test recorder that helps in writing tests.

\section{SoapUI}

SoapUI is a cross-platform functional testing tool. It has been specifically designed to help automatically test APIs such as SOAP and REST interfaces to ensure the interoperability of different application.

\section{QuickTest Professional}

QuickTest Professional (QTP) helps the tester to perform an automated functional testing. It supports only window XP and 
developed only in VBScript or JavaScript. With QTP it is easy to edit the script, playback and validate the results.

\subsection{Conclusion}

This paper presents a study on various automated web testing tools that used on different platforms. Automated testing tools helps the tester to easily automate the whole testing process. Automation testing improves the accuracy and also save time of the tester as compared to the manual testing.

\section{References}

[1] Leelavathi Rajamanickam, "Testing Tool for Object Oriented Software" International Journal of scientific research and management (IJSRM), vol 2. Issue 8 august 2014, pages: 1205-1208 (2014).

[2] Richa Rattan, "Comparative Study Of Automation Testing Tools: Quick Test Professional \& Selenium”, Vsrd International Journal Of Computer Science \& Information Technology, Vol. 3 No. 6 June 2013.

[3] Manjit Kaur, Raj Kumar, "Comparative Study of Automated Testing Tools: Test Complete and Quick Testpro", International Journal of Computer Applications (0975-8887) Vol 24 No. 1, June 2011.

[4] Manual Testing Http://En.Wikipedia.Org/Wiki/Manual Testing

[5] Leckraj Nagowah and Purmanand Roopnah, "Ast -A Simp Le Automated System Testing Tool”, IEEE, 978-1- 4244-5540-9/10, 2010

[6] S Thummalapenta, S Sinha, N Singhania, S Chandra, "Automating Test Automation", Proceedings Of The 34th International Conference On Software Engineering (ICSE), May 2012

[7] Test Automation:

Http://Researcher.Watson.Ibm.Com/Researcher/View_Group.Php?Id $=3208$ 
[8] Zhi Quan Zhou, Bernhard Scholz, Giovanni Denaro, “Automated Software Testing and Analysis: Techniques, Practices and Tools", Proceedings of the 40th Annual Hawaii International Conference on System Sciences (Hicss'07) 0-7695-2755-8/07. 